\title{
Intracranial hypotension due to lumbar puncture presenting as a pseudo-subarachnoid hemorrhage on imaging
}

\begin{abstract}
Background: A radiological pseudo-subarachnoid hemorrhage (pseudo-SAH) is defined as an increased attenuation along the tentorium cerebelli and falx, the basal cisterns, the Sylvian fissures and the subarachnoid spaces resembling a subarachnoid hemorrhage (SAH) on brain computed tomography (CT) scan. Several causes of pseudo-SAH have been reported. We present a female patient with headache and intracranial hypotension due to lumbar puncture (LP) in which the CT scan showed similar features to a typical SAH. The adequate diagnosis and treatment of intracranial hypotension was delayed until CT angiography (CTA), digital subtraction angiography (DSA) and magnetic resonance images (MRI) were performed to rule out SAH and its potential cause.

Case presentation: We present a 29 -year-old woman who suffered from a severe postlumbar puncture headache (PLPH). A non-contrast brain CT scan revealed blurred frontal and parietal right sulci, and increased attenuation of the ipsilateral Sylvian fissure. SAH was diagnosed but the results of CTA and DSA were negative. A brain MRI demonstrated right brain sagging. Intracranial hypotension due to LP presenting as a pseudo-SAH was finally diagnosed, and her headache subsided after conservative therapy.

Conclusion: In patients with PLPH, intracranial hypotension should be considered in the differential diagnosis between SAH and pseudo-SAH. Although these two entities can share some clinical and radiologic features; intracranial hypotension has some distinctive MRI and CT findings. Brain sagging, bilateral subdural effusions or hematoma and pachymeningeal enhancement on MRI may be valuable findings for distinguishing between intracranial hypotension and SAH. These findings should be recognized to avoid unnecessary invasive procedures, delays in the diagnosis and implementing an adequate treatment.
\end{abstract}

Keywords: post-lumbar puncture headache, pseudo-subarachnoid hemorrhage, intracranial hypotension
Volume 5 Issue 2 - 2018

\author{
Paola A Barón Ródiz,' Concepción Ferreiro \\ Argüelles,' Judit Diaz Diaz, ${ }^{2}$ Alice Pérez De \\ Los Ríos' \\ 'Department of Radiology, Hospital Universitario Severo Ochoa, \\ Spain \\ ${ }^{2}$ Department of Neurology, Hospital Universitario Severo \\ Ochoa, Spain
}

\begin{abstract}
Correspondence: Paola A Barón Ródiz, Department of Radiology, Hospital Universitario Severo Ochoa, Madrid, Spain, Tel 0034-9|4-8|8-000, Fax 0034-9|6-940-7|7, Email paobaron12@gmail.com
\end{abstract}

Received: February 27, 2018 | Published: April 03, 2018
Abbreviations: PLPH, post-lumbar puncture headache; CSF, cerebrospinal fluid; CT, computerized tomography; LP, lumbar puncture; SAH, subarachnoid hemorrhage; CTA, computerized tomography angiography; DSA, digital subtraction angiography; MRI, magnetic resonance images

\section{Background}

Post-lumbar puncture headache (PLPH) is one of the most common complications of this procedure. It occurs in 10 to 30 percent of patients due to leakage of cerebrospinal fluid (CSF) from the dura mater with traction of pain-sensitive structures. ${ }^{1,2}$ It characteristically presents with severe orthostatic frontal or occipital headache $(15 \%)$ within 6 to 72 hours from the procedure, is exacerbated with the upright position and improved in the supine position. ${ }^{3,4}$ Common risk factors include: female gender, a prior history of headache, age 20 to 50 years, and bevel orientation perpendicular to the longitudinal fibers of the dura. ${ }^{4-6}$ Two other factors had been thought to increase the risk of developing PLPH: large amounts of fluid removed and the experience of the physician performing the procedure.

Without treatment, the headache typically lasts 2 to 15 days. Most PLPHs are typically mild and resolve spontaneously. Conservative therapies (bed rest and oral analgesics, including opioids) are generally recommended for the first 24 hours. For patients with moderate to severe prolonged headache ( $>24$ hours) and / or refractory to conservative measures, epidural blood patch is suggested. ${ }^{7,8}$

A non-contrast brain computerized tomography (CT) scan is the first imaging procedure performed in patients that suffer from acute headache, especially after a lumbar puncture (LP), and it might be normal in around $20 \%$ of the case. ${ }^{9,10}$ Brain CT manifestations of intracranial hypotension include: brain sagging, bilateral subdural effusions or hematoma, increased tentorial enhancement, slit shaped ventricles associated with tight basal cisterns and scant CSF over the cortex. ${ }^{11}$ On magnetic resonance images (MRI) diffuse pachymeningeal enhancement and dural venous dilatation may be seen. ${ }^{12}$ In addition to the clinical presentation and imaging studies, the diagnosis of intracranial hypotension requires a CSF pressure measurement by lumbar tapping of less than $100 \mathrm{~mm} \mathrm{H}_{2} \mathrm{O} \cdot{ }^{13}$

Intracranial hypotension may mimic subarachnoid hemorrhage (SAH) in several ways including clinical symptoms, radiological features and CSF findings. Thunderclap headache, nausea, vomiting, posterior neck pain and nuchal rigidity are present in both of them; therefore, these symptoms are not useful for distinguishing between 
intracranial hypotension and SAH. ${ }^{14}$ Moreover, LP and CSF study cannot distinguish between these two entities because a normal CSF pressure may be present in certain patients with intracranial hypotension and xanthochromia from a traumatic spinal tap may also lead to misdiagnosis (traumatic LP is frequent in patients with intracranial hypotension because of the low CSF pressure and the difficulty in performing the tap). ${ }^{9,13}$

Also, intracranial hypotension may mimic SAH on brain CT scan ("pseudo-SAH"). A pseudo-SAH is defined as an increased attenuation along the tentorium cerebelli and falx, the basal cisterns, the Sylvian fissures and the subarachnoid spaces resembling a SAH on brain CT scan. The pathophysiology of pseudo-SAH on brain CT scan may be explained by the following theory: ${ }^{15}$ the brain sagging may compress the basal cisterns, the Sylvian fissures and the subarachnoid spaces, and subsequently compromise the CSF circulation. It results in brain edema and obliteration of these three spaces. In addition, intracranial hypotension also induces the dilatation of cerebral veins and dural venous sinuses secondary to the compensation for decreased CSF volume, which accounts for the increased attenuation observed on CT scan along the basal cisterns, the Sylvian fissures and the subarachnoid spaces.

Several causes of pseudo-SAH have been reported, some of them are: ${ }^{16-20}$ encephalopathy with brain swelling (hyponatremia, anoxicischemic and metabolic encephalopathy), pyogenic leptomeningitis, leptomeningeal carcinomatosis, venous sinus thrombosis, status epilepticus, intracranial hypotension (spontaneous or due to lumbar puncture), hyperperfusion syndrome or luxury perfusion, polycythemia, oxygen therapy or inhaled anesthetics, leakage of highdose intravenous contrast medium into the subarachnoid space and / or intrathecally administered contrast material. We present a female patient with headache and intracranial hypotension due to LP in which the CT scan showed similar features to a typical SAH. The adequate diagnosis and treatment of intracranial hypotension was delayed until CT angiography (CTA), digital subtraction angiography (DSA) and MRI were performed.

\section{Case presentation}

A 29-year-old woman with no medical history of interest suffered from intermittent visual loss related to movements (as wake up from bed or bend down) for the last 4 months. She also complained about an arcuate defect. She was assessed by an ophthalmologist who detected a functional deficit in the vision of the right eye with bilateral optic disc swelling, so she was referred to the neurologist. Physical and neurological examinations were unremarkable.

A first brain CT scan was normal. A LP was performed and it revealed that the CSF pressure was $470 \mathrm{~mm} \mathrm{H}_{2} \mathrm{O}$; the appearance of the CSF was clear without pleocytosis, red blood cells or xanthochromia. After taking out $15 \mathrm{ml}$ of CSF, the pressure went down to $120 \mathrm{~mm}$ $\mathrm{H}_{2} \mathrm{O}$ and she was sent home. Two days later, she was brought to the emergency room because of persistent orthostatic headache since the LP was performed. The headache was intense, oppressive and was located in the frontal area. It could be slightly relieved by lying down whereas it was worse in the upright position. The headache was not accompanied by nausea or vomiting, and there was no improvement with acetaminophen. She denied a history of trauma, surgery, spinal anesthesia, or other medical illnesses.
The initial vital signs and neurological examination were normal. A second brain $\mathrm{CT}$ scan revealed blurred frontal and parietal right sulci, and increased attenuation of the ipsilateral Sylvian fissure; so SAH convexity pattern (grade I- Modified Fisher scale-) was diagnosed (Figure 1). Brain CTA and DSA were performed during hospitalization but no vascular malformations or aneurysms were found (Figure 2). Non-aneurysmal SAH was diagnosed. A brain MRI performed demonstrated right brain sagging and no signs of SAH were seen on FLAIR and susceptibility weighted images (Figure 3). Intracranial hypotension due to LP presenting as a pseudo-SAH was finally diagnosed. The patient's headache improved after conservative therapy (bed rest and intravenous fluid supplement).

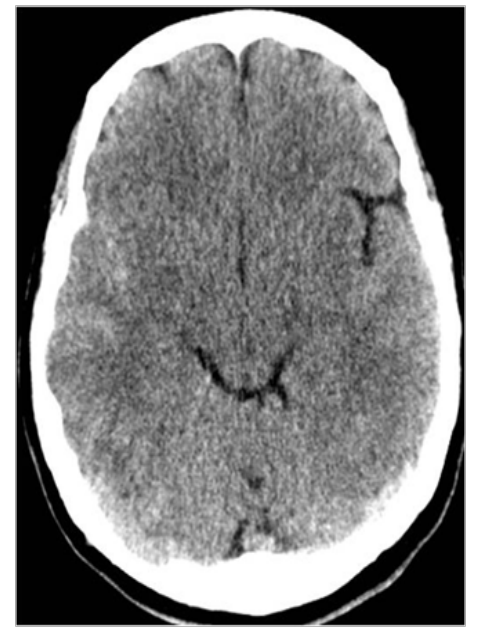

Figure I Brain CT scan performed in the emergency room due to severe post-lumbar puncture headache. Axial non-contrast brain CT image shows blurred fronto-parietal right sulci and increased attenuation of the ipsilateral Sylvian fissure resembiling subarachnoid hemorrhage convexity pattern.

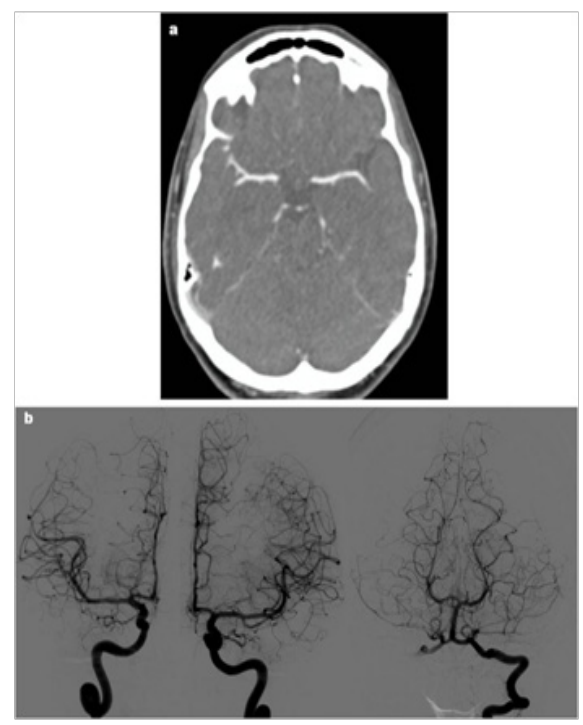

Figure 2 Brain CT angiography (CTA) and Digital subtraction angiography (DSA) performed during hospitalization. No vascular malformations and / or aneurysms were found on (a) CTA and (b) DSA. 


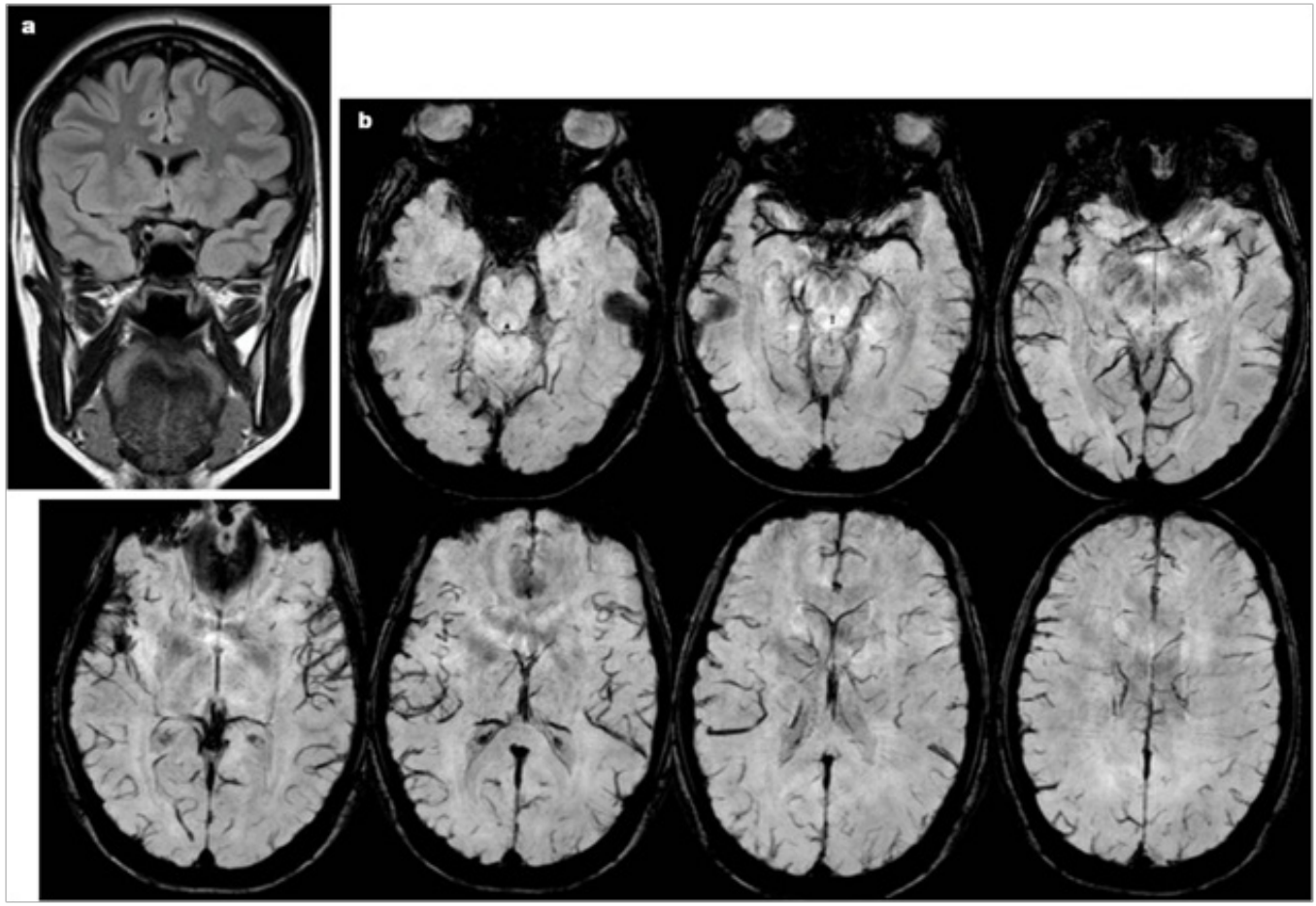

Figure 3 Brain MRI performed during hospitalization.

Figure 3 (A) Coronal FLAIR weighted image demonstrates right brain sagging with downward displacement and narrowing of the subarchnoid space of the ipsilateral Sylvian fissure with no signs of subarachnoid hemorrhage. Figure $\mathbf{3}$ (B) Axial susceptibility weighted images in caudo-cranial direction show no hemorrhagic areas.

\section{Discussion}

SAH was hard to rule out because of the severe headache and $\mathrm{CT}$ findings. Furthermore, the presentation of pseudo-SAH on brain CT (increased attenuation of the frontal and parietal right sulci and the ipsilateral Sylvian fissure) made an accurate diagnosis more complicated. However, negative results for vascular aneurysms and / or malformations on CTA and DSA excluded the diagnosis of SAH.

Post-puncture headache, the appearance of brain sagging on brain MRI and symptoms relieved by recumbency and fluid supplements were the clues for diagnosing post-puncture intracranial hypotension. In this case, we believe that the amount of fluid removed caused an abrupt descent of the CSF pressure, exceeding the rate of CSF production and resulting in low CSF volume. ${ }^{2}$ In that order, we believe that the brain sagging narrowed the right subarachnoid spaces and displaced the CSF. Then, subarachnoid spaces became devoid of the hypoattenuated CSF and were filled by larger fractions of meninges and blood vessels that, increasing their $\mathrm{CT}$ attenuation, contributed to the pseudo-SAH appearance. ${ }^{21}$

In some cases, the measure of attenuation values within the basal cisterns or the spaces affected, which are usually lower than in patients with SAH, in whom attenuation values typically range from 60 to 70 $\mathrm{HU}$, might allow pseudo-SAH recognition and obviate more invasive testing. ${ }^{21-23}$

Excluding head injury, SAH leads to the performance of angiographic studies to rule out vascular lesions. Thus, the main point of this case is the importance of recognizing the signs of pseudo$\mathrm{SAH}$ on brain $\mathrm{CT}$ in patients with clinical suspicion of intracranial hypotension, especially in the emergency room, to avoid invasive testing, delays in the diagnosis and delays implementing an effective treatment.

\section{Conclusion}

On imaging studies intracranial hypotension may be seen similar to $\mathrm{SAH}$-the so called pseudo-SAH pattern. This imaging finding may lead to misinterpretation, raising the suspicion of an underlying source of bleeding. Therefore, it is important to raise awareness of this type of presentation to avoid unnecessary invasive procedures that would delay the correct diagnosis and the adequate treatment.

\section{Acknowledgements}

None.

\section{Funding}

Not applicable

\section{Author's contributions}

PABR designed and performed the case, with the help of CFA, JDD, and APDR. All authors read and approved the final manuscript. 


\section{Competing interests}

None of the authors have any competing interests in the manuscript.

\section{Ethics approval and consent to participate}

Not applicable

\section{Consent for publication}

Written informed consent was obtained from the patient for publication of this case report and accompanying images.

\section{Availability of data and materials}

Not applicable

\section{References}

1. Raskin NH. Lumbar puncture headache: a review. Headache. 1990;30(4):197-200.

2. Fernández E. Headaches associated with low spinal fluid pressure. Headache. 1990;30(3):122-128.

3. Silberstein SD, Marcelis J. Headache associated with changes in intracranial pressure. Headache. 1992;32(2):84-94.

4. Amorim JA, Gomes de Barros MV, Valença MM. Post- dural (post- lumbar) puncture headache: risk factors and clinical features. Cephalalgia. 2012;32(12):916-923.

5. Kuntz KM, Kokmen E, Stevens JC, et al. Post- lumbar puncture headaches: experience in 501 consecutive procedures. Neurology. 1992;42(10):1884-1887.

6. Evans RW, Armon C, Frohman EM, et al. Assessment: prevention of post- lumbar puncture headaches: report of the therapeutics and technology assessment subcommittee of the american academy of neurology. Neurology. 2000;55(7):909-914.

7. Boonmak P, Boonmak S. Epidural blood patching for preventing and treating post- dural puncture headache. Cochrane Database Syst Rev. 2010;20(1):CD001791.

8. Mokri B. Low cerebrospinal fluid pressure syndromes. Neurol Clin 2004;22(1):55-74.

9. Schievink WI, Wijidicks EF, Meyer FB, et al. Spontaneous intracranial hypotension mimicking aneurysmal subarachnoid hemorrhage. Neurosurgery. 2001;48(3):513-516.
10. Ferrante E, Savino A. Thunderclap headache caused by spontaneous intracranial hypotension. Neurol Sci. 2005;26(2):155-157.

11. Murros K, Fogelholm R. Spontaneous intracranial hypotension with slit ventricles. J Neurol Neurosurg Psychiatry. 1983;46(12):1149-1151.

12. Sainani NI, Lawande MA, Pungavkar SA, et al. Spontaneous intracranial hypotension: a study of six cases with MR findings and literature review. Australasian Radiol. 2006;50(5):419-423.

13. Miyazama K, Shiga Y, Hasegawa T, et al. CSF hypovolemia vs intracranial hypotension in "spontaneous intracranial hypotension syndrome”. Neurology. 2003;60(6):941-947.

14. Schievink WI. Misdiagnosis of spontaneous intracranial hypotension. Arch Neurol. 2003;60(12):1713-1718.

15. Schievink WI, Maya M, Tourje J, et al. Pseudo- subarachnoid hemorrhage: a CT- finding in spontaneous intracranial hypotension. Neurology. 2005;65(1):135-137.

16. Chun Yu L, Ping Hong L, Jui Hsun F, et al. Pseudo- Subarachnoid Hemorrhage: A Potential Imaging Pitfall. Can Assoc Radiol J. 2014;65(3):225-231

17. Eckel T, Breiter SN, Lee HM. Subarachnoid contrast enhancement after spinal angiography mimicking diffuse subarachnoid hemorrhage. AJR Am J Roentgenol. 1998;170(2):503-505.

18. Mendelsohn D, Moss ML, Chason DP, et al. Acute purulent leptomeningitis mimicking subarachnoid hemorrhage on CT. J Comput Assist Tomogr. 1994;18(1):126-128.

19. Spiegel M, Fox AJ, Vinuela F, et al. Increased density of tentorium and falx: a false positive CT sign of subarachnoid hemorrhage. Can Assoc Radiol J. 1986;37(4):243-247.

20. Osborn AG. Diagnostic neuroradiology. St. Louis, MO: Mosby. 1994; p. 212.

21. Ferrante E, Regna Gladin C, Arpino I, et al. Pseudo subarachnoid hemorrhage: a potential imaging pitfall associated with spontaneous intracranial hypotension. Clin Neurol Neurosurg. 2013;115(11):2324 2328.

22. Ohkawa M, Masatada T, Toyama Y, et al. CT angiography with helical $\mathrm{CT}$ in assessment of acute stage of subarachnoid hemorrhage. Radiat Med. 1998;16(2):91-97.

23. Fujita S. Computed tomographic grading with Hounsfield number related to delayed vasospasm in cases of ruptured cerebral aneurysm. Neurosurgery. 1985;17(4):609-612. 